Wie jemand denkt, verraten seine Metaphern. Daher lohnt oft ein verlängerter Forschungsaufenthalt im Urwald der Metaphern, die sich - wenn Hans Blumenberg nicht fehlgeht - in philosophicis als ,absolute Metaphern“2 ausprägen können, also als Metaphern, die sich nicht ohne Substanzverlust in eine Begriffssprache übersetzen lassen, ja sich einer Reduktion auf Begriffliches überhaupt entziehen. ${ }^{3}$ Metaphern haben ihre Schicksale und Konjunkturen.

Hoch gehandelt wird gegenwärtig die Metapher einer Architektur des Wissens. Einerseits gründet diese Hausse auf einer neuen Empfänglichkeit für das Selbstverständnis von Wissenschaft in der Vormoderne, die sich oft genug die feste Fügung von Gebäuden zum Strukturmodell wählte: Das Haus des Wissens hat ein sicheres Fundament, unerschütterliche Mauern, ein gedecktes Dach, Türen mit strenger Eingangskontrolle und Fenster mit mehr oder weniger Ausblick (gelegentlich hat es auch, wie das Rathaus von Schilda, weder Türen noch Fenster). Andererseits drückt sich in der Hausse der Wissensarchitekturforschung auch eine Sehnsucht von heute Forschenden aus, sinnenfällig geworden in der Metapher vom Gebäude des Wissens - und es ist stets eher ein Schloss als eine Hütte: Was uns fehlt und was viele von uns insgeheim gerne hätten, ist eine festgefügte Ordnung festgegründeten Wissens. Wenn wir diesen Mangel schon nicht durch unsere eigene Geistesbautätigkeit ausgleichen können, so ist doch der Blick auf überlieferte Bauten dazu angetan, uns über diesen Mangel zu trösten.

Schon die Wissensakteure des mittleren und späteren 19. Jahrhunderts taten sich freilich schwer, als Wissensarchitekten aufzutreten. Wenn Friedrich Nietzsche sein Denken nicht mehr zu einem unerschütterlichen Gebäude fügt, so ist er in dieser Nicht-Fügung durchaus ein typischer Repräsentant seiner Epoche. Dennoch gewinnt bei ihm die Architektur auch als Reservoir von Denkfiguren eine gewisse Prominenz. Dies behandelt an einem konkreten Beispiel der erste Abschnitt. Der zweite hingegen beschäftigt sich mit Nietzsches Versuchen, alle feste Fügung zu zerbrechen und die Vorstellung von Wissen als Architekturwerk ins Reich der frommen Fiktion zu verbannen.

1 Für die kritische Lektüre dieses Textes danke ich Frau Julia Maas.

2 Vgl. Hans Blumenberg, Paradigmen zu einer Metaphorologie, Frankfurt a. M. 1999, 12.

3 Dazu ausführlich die Beiträge in Ralf Konersmann (Hg.), Wörterbuch der philosophischen Metaphern, Darmstadt 2007. 


\section{Nietzsches Architekturen}

Nietzsches Nachdenken über die bauliche Gestaltung der Wirklichkeit, sein Interesse an Fragen der Architekturtheorie und Architekturpraxis hat in jüngster Zeit vermehrt die Aufmerksamkeit auf sich gezogen. Neben einigen Aufsätzen widmen sich drei Monographien Nietzsches Verhältnis zur Architektur ${ }^{4}$, angefangen mit seinen über Richard Wagner vermittelten Lektüren von Gottfried Sempers Schriften ${ }^{5}$ und seiner intensiven Auseinandersetzung mit Jacob Burckhardts kunsthistorischen Architekturanalysen über seine reale Begegnung mit architektonischen Meister- und Minderwerken, namentlich in Italien ${ }^{6}$, sowie über seine Einlassungen zu den Übergängen von Musik zu Architektur im Falle Wagners ${ }^{7}$, gipfelnd schließlich in seiner Begeisterung für Alessandro Antonellis Mole in Turin, die er noch ganz am Ende seines bewussten Lebens thematisiert: Im Brief an Peter Gast vom 30. Dezember 1888 stellt er das Riesengebäude dem Zarathustra zur Seite und will es „Ecce homo getauft“ haben (KSB 8, 565 f., Nr. 1227), um dann im allerletzten Brief am 6. Januar Burckhardt zu bescheiden: „Antonelli war ich selbst“ (ebd., 579, Nr. 1256). ${ }^{8}$

Nietzsches Denken als solches hat wiederum seit der vorletzten Jahrhundertwende viele Architekten inspiriert. ${ }^{9}$ Namentlich seine Reflexionen über die Wirkung

4 Markus Breitschmid, Der bauende Geist. Friedrich Nietzsche und die Architektur, Luzern 2001; Fritz Neumeyer, Der Klang der Steine. Nietzsches Architekturen, Berlin 2001. Zu beiden Büchern die Rezension von Matthias Müller in: Nietzsche-Studien 32 (2003), 526-530; Jörg H. Gleiter, Der philosophische Flaneur. Nietzsche und die Architektur, Würzburg 2009. Dazu die Rezension von Renate Reschke in: Nietzscheforschung 17 (2010), 332-336. Vgl. Alexandre Kostka, Irving Wohlfarth (Hg.) Nietzsche and „An Architecture of Our Minds“, Los Angeles 1999.

5 Siehe dazu neben Fritz Neumeyer, Der Klang der Steine, auch Hans-Georg von Arburg, „Freier Faltenwurf des Gemüths“. Zu einem Stilideal in der Architekturästhetik Gottfried Sempers und Friedrich Nietzsches, in: Sabine Schneider, Heinz Brüggemann (Hg.), Gleichzeitigkeit des Ungleichzeitigen. Formen und Funktionen von Pluralität in der ästhetischen Moderne, München 2011, 105-124.

6 Hierzu ist besonders einschlägig Tilmann Buddensieg, Nietzsches Italien. Städte, Gärten und Paläste, Berlin 2002.

7 Wagner fürchte „die Versteinerung, die Krystallisation, den Uebergang der Musik in das Architektonische, - und so stellt er dem zweitactigen Rhythmus einen dreitactigen entgegen, führt nicht selten den Fünf- und Siebentact ein, wiederholt die selbe Phrase sofort, aber mit einer Dehnung, dass sie die doppelte und dreifache Zeitdauer bekommt“ (VM, KSA 2, 435).

8 Eine nützliche Zusammenstellung architekturrelevanter Nietzsche-Stellen in chronologischer Ordnung bietet Markus Breitschmid, Der bauende Geist, 127-204, freilich gerade unter Ausklammerung der Briefe.

9 Auch dies ist Gegenstand vielfältiger Forschungsanstrengungen. Exemplarisch seien drei Aufsätze genannt: Peter Bernhard, „Ich-Überwindung muß der Gestaltung vorangehen“. Zur Nietzsche-Rezeption des Bauhauses, in: Andreas Urs Sommer (Hg.), Nietzsche - Philosoph der Kultur(en)?, Berlin, New York 2008, 273-282; Tilmann Buddensieg, Also baute Zarathustra, in: Marco Brusotti, Renate Reschke (Hg.), „Einige werden posthum geboren“. Friedrich Nietzsches Wirkungen, Berlin, Boston 2012, 227-252; Henning Ottmann, Zarathustra - Spuren in Architektur, bildender Kunst und Malerei, in: Ma- 
von Architektur auf Menschen haben als eine Art Handreichung zur architektonischen Wirklichkeitsgestaltung verstanden werden können; beispielsweise, wenn er unter dem Titel Architektur der Erkennenden empfiehlt: „Es bedarf einmal und wahrscheinlich bald einmal der Einsicht, was vor Allem unseren grossen Städten fehlt: stille und weite, weitgedehnte Orte zum Nachdenken, Orte mit hochräumigen langen Hallengängen für schlechtes oder allzu sonniges Wetter, wohin kein Geräusch der Wagen und der Ausrufer dringt und wo ein feinerer Anstand selbst dem Priester das laute Beten untersagen würde: Bauwerke und Anlagen, welche als Ganzes die Erhabenheit des Sich-Besinnens und Bei-Seitegehens ausdrücken“ (FW, KSA 3, 524). Wer freilich, wie Nietzsche, ein waches Auge für die architektonische ,Mit'bedingtheit menschlicher Existenz hat und deshalb danach strebt, die architektonischen Bedingungen zu optimieren, unter denen Menschen ihr Dasein fristen - beispielsweise mittels einer Architektur, die durch Freiräume Denkräume eröffnet - , muss deshalb bei der Decodierung der kulturellen Welt noch nicht zwangsläufig der Architektur eine Schlüsselrolle einräumen: Es könnte ja durchaus sein, dass der Faktor Architektur wegen ihrer kolossalen Sichtbarkeit in ihrer Bedeutung für das menschliche Leben und konkret für die Möglichkeiten des Philosophierens überschätzt wird, auch wenn man unstrittig im Spiegelsaal eines Schlosses anders philosophieren wird als in dessen Kerkerverließ. Das wache Auge für die architektonische Mitbedingtheit menschlicher Existenz könnte auch dazu führen, der Architektur keine Macht über das Denken einzuräumen und mit Bedacht zu vermeiden, aus der Architektur die Metaphern und Denkfiguren zu beziehen, sich dagegen wehrend, dass die Muster architektonischer Wirklichkeitsgestaltung das Denken kolonialisieren.

Muss es nicht bedenklich stimmen, dass ausgerechnet ein so technikkritischer Denker wie Martin Heidegger dem Haus, bei Lichte besehen dem Gestell par excellence, in seiner postmetaphysischen Gedankenbildung einen so prominenten Platz einräumt? „Die Sprache ist das Haus des Seins. In ihrer Behausung wohnt der Mensch. Die Denkenden und Dichtenden sind die Wächter dieser Behausung. "10 Gewiss, das Haus in größtmöglicher Allgemeinheit ist für die Lebensart der Menschen konstitutiv, seit sie von den Bäumen heruntergeklettert und aus den Höhlen herausgekrochen sind. Aber das Haus steht nicht nur für Sesshaftigkeit und Wohnen, sondern auch für Abschottung, für die Trennung von Innen und Außen, für Horizontbeschränkung. Soll das Sein tatsächlich in der Sprache eingezäunt, in ihr eingemauert werden? Vor dem Heidegger-Exegeten gähnt ein Abgrund, denn erliegt der Meister, hier erklärtermaßen noch die „Sprache der Metaphysik“ sprechend ${ }^{11}$, nicht schlicht der Verführungskraft jener alten Metapher, wonach die Wirklichkeit wie ein Haus gestaltet ist,

thias Mayer (Hg.), Also wie sprach Zarathustra? West-östliche Spiegelungen im kulturgeschichtlichen Vergleich, Würzburg 2006, 217-242.

10 Martin Heidegger, Über den Humanismus, Frankfurt a. M. 1949, 5.

11 Ebd. 
das das Denken als Wissensarchitektur zu reproduzieren hat? Warum dem Sein nicht die Offenheit der Unbehaustheit lassen?

Nietzsche seinerseits hütet nicht das Sein, sondern sich vor der Versuchung, die Wirklichkeit architektomorph zu verstehen. Das trifft selbst für jene Passagen im Spätwerk zu, die der Architektur einen zentralen Stellenwert einräumen, ja ihre Ausübung als höchste Kunst, nämlich als Willenskunst feiern. ${ }^{12}$ In einer vorbereitenden Aufzeichnung zu den späteren Abschnitten 10 und 11 der Streifzüge eines Unzeitgemässen in der Götzen-Dämmerung werden verschiedene Künste im Hinblick auf die Frage betrachtet: „Was bedeutet der Gegensatz ,dionysisch“ und ,apollinisch“, beide als Arten des Rausches verstanden? Letzteres hat vor allem das Auge erregt: so daß es die Kraft der Vision bekommt. Ersteres erregt das gesammte Affekt-System: so daß es die Kraft der Transfiguration, Darstellens, Verwandlung, Schauspielerei und Tanzbewegung heraustreibt ... das Wesentliche ist die Kraft der Metamorphose: so daß der Affekt leicht angedeutet, sofort zur Realität fortgeht ...“ (KGW IX 8, W II 5, 165).

Dabei wird nach dem Musiker und dem Lyriker auch vom Architekten Rechenschaft gefordert, wobei dessen Zuordnung zum jeweiligen Rauschtypus unterbestimmt bleibt:

\begin{abstract}
„〈D $\rangle$ er Architekt stellt eine große Nützlichkeit in ihrer überzeugendsten und stolzesten Form dar[...]: in dem Bau soll sich die Macht, der Wille zur Macht versichtbaren. Eine Beredsamkeit der Seele in großen Linien ... “ (ebd.). Nietzsche hat diese Passage im Manuskript wie folgt überarbeitet: „,D〉er Architekt stellt eine〈n〉 großen Willensakt dar, den Rausch eines großen Willens in ihrer [sic] überzeugendsten und stolzesten Form [...]: in dem Bau soll sich die Macht, der Wille

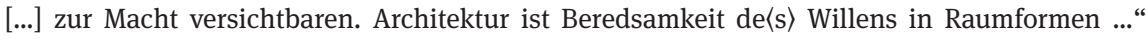
(ebd.).
\end{abstract}

Erst die Druckfassung in den Streifzügen eines Unzeitgemässen 11 der Götzen-Dämmerung stellt unmissverständlich klar, dass die Architektur als Kunst weder dem Bereich des Apollinischen noch des Dionysischen zuzuordnen ist: „Der Architekt stellt weder einen dionysischen, noch einen apollinischen Zustand dar: hier ist es der grosse Willensakt, der Wille, der Berge versetzt, der Rausch des grossen Willens, der zur Kunst verlangt. Die mächtigsten Menschen haben immer die Architekten inspirirt; der Architekt war stets unter der Suggestion der Macht. Im Bauwerk soll sich der Stolz, der Sieg über die Schwere, der Wille zur Macht versichtbaren; Architektur ist eine Art Macht-Beredsamkeit in Formen, bald überredend, selbst schmeichelnd, bald bloss befehlend. Das höchste Gefühl von Macht und Sicherheit kommt in dem zum Ausdruck, was grossen Stil hat. Die Macht, die keinen Beweis mehr nöthig hat; die es verschmäht, zu gefallen; die schwer antwortet; die keinen Zeugen um sich fühlt; die

12 Vgl. zum Folgenden Andreas Urs Sommer, Kommentar zu Nietzsches Der Fall Wagner. Götzen-Dämmerung = Historischer und kritischer Kommentar zu Friedrich Nietzsches Werken, hg. von der Heidelberger Akademie der Wissenschaften, Bd. 6/1, Berlin, Boston 2012, 434-443. 
ohne Bewusstsein davon lebt, dass es Widerspruch gegen sie giebt; die in sich ruht, fatalistisch, ein Gesetz unter Gesetzen: Das redet als grosser Stil von sich. - “ (GD, KSA 6, 118 f.).

Bei der Behandlung der Kunst- und Künstler-Typen in den Abschnitten 10 und 11 der Streifzüge eines Unzeitgemässen blendet Nietzsche ausgerechnet jenen Künstler aus, den er in der Geburt der Tragödie (vgl. GT, KSA 1, 30) als verkörperte Synthese des Dionysischen und des Apollinischen eingeführt hatte, nämlich den TragödienKünstler. Die Götzen-Dämmerung weist verschiedene Typen des Dionysischen und des Apollinischen aus, jedoch keine synthetischen Formen, die Nietzsche einst als höchste Künstler galten. Noch mehr dürfte überraschen, dass der Architekt, allein vom Willen berauscht, einen Künstlertypus jenseits der Sphären des Apollinischen und Dionysischen darstellen soll. Offensichtlich erscheint Nietzsche sein altes, ursprünglich auf Richard Wagner gemünztes binäres Schema Dionysisch/Apollinisch nicht mehr hinreichend, um die Breite und Fülle künstlerischer Äußerungen abzudecken; es gibt noch Anderes, Höheres außerhalb dieses Schemas. Die Ausführungen über den Architekten dienen sichtlich dazu, die von Nietzsche früher etablierte Hierarchie der Künste mit der tragischen Kunst an oberster Stelle zu unterminieren, indem jetzt eine eher unterbelichtete Kunst, eben die Baukunst, an die oberste Stelle tritt. Die strategische Motivation zu dieser Umbesetzung liegt auf der Hand: Die vom décadence-Künstler Wagner in Personalunion repräsentierten Disziplinen Musik und Schauspielerei dürfen nicht länger den prominentesten Platz besetzen, weil sonst wiederum Wagner als deren größter Meister erscheinen könnte - obwohl Nietzsche im Fall Wagner dessen Meisterschaft jüngst erst vehement bestritten hatte.

Aufschlussreich ist die Transformation der Überlegungen in den beiden Manuskriptfassungen von W II 5 zur Druckfassung in der Götzen-Dämmerung nicht nur, weil erst letztere den Architekten ausdrücklich aus der Gebundenheit an den apollinischen oder dionysischen Rausch ausnimmt. In der ersten Fassung tauchen zwar bereits die Stichworte „Macht“ und „Wille zur Macht“ auf, mit ihnen zugleich aber auch noch ein Epitheton, das traditionell mit Architektur liiert ist, nämlich die ,große Nützlichkeit“ (KGW IX 8, W II 5, 165): Baukunst ist ja (fast) nie reiner Selbstzweck, sondern schafft etwas, was Menschen auch jenseits der Kunst brauchen: Behausung. In der zweiten Manuskriptversion (sowie im Druck) bleibt diese „Nützlichkeit“ jedoch außen vor; sie wird zunächst unmittelbar ersetzt durch „eine〈n〉 großen Willensakt“; jetzt weicht sie dem „Rausch eines großen Willens“ (KGW IX 8, W II 5, 165), ohne jegliche Spezifikation, ob dies denn für jeden biederen Baumeister eines Bauernhofes oder einer Badeanstalt gelten soll - auch wenn man vermuten darf, dass da für Nietzsche kein Willensrausch am Werke ist. Zu einem eigentlichen Willenskunstprogramm weiten sich die Überlegungen zur Architektur dann erst in der Druckfassung aus.

Erhellend ist der Vergleich dieser Version mit einschlägigen Äußerungen Arthur Schopenhauers, der ein Vorreiter philosophischer Architektur-Reflexion gewesen ist: „Wenn wir nun die Baukunst, bloß als schöne Kunst, abgesehn von ihrer Bestimmung zu nützlichen Zwecken, in welchen sie dem Willen, nicht der reinen Erkenntniß dient 
und also nicht mehr Kunst in unserm Sinne ist, betrachten; so können wir ihr keine andere Absicht unterlegen, als die, einige von jenen Ideen, welche die niedrigsten Stufen der Objektität des Willens sind, zu deutlicher Anschaulichkeit zu bringen: nämlich Schwere, Kohäsion, Starrheit, Härte, diese allgemeinen Eigenschaften des Steines, diese ersten, einfachsten, dumpfesten Sichtbarkeiten des Willens, Grundbasstöne der Natur; und dann neben ihnen das Licht, welches in vielen Stücken ein Gegensatz jener ist. Selbst auf dieser tiefen Stufe der Objektität des Willens sehn wir schon sein Wesen sich in Zwietracht offenbaren: denn eigentlich ist der Kampf zwischen Schwere und Starrheit der alleinige ästhetische Stoff der schönen Architektur: ihn auf mannigfaltige Weise vollkommen deutlich hervortreten zu lassen, ist ihre Aufgabe. Sie löst solche, indem sie jenen unvertilgbaren Kräften den kürzesten Weg zu ihrer Befriedigung benimmt und sie durch einen Umweg hinhält, wodurch der Kampf verlängert und das unerschöpfliche Streben beider Kräfte auf mannigfaltige Weise sichtbar wird. “13

Schopenhauer exponiert also wie Nietzsche in der ersten Überlegung von W II 5 ebenfalls an erster Stelle das Moment der lebenspraktischen Nützlichkeit, um dann aber entschlossen von ihr abzusehen und Architektur als ,schöne Kunst' zu betrachten. Dabei kommt ihr aber, weil sie mit den untersten Verkörperungsformen des Willens beschäftigt ist, auch ein niedriger Stellenwert zu. Nietzsche könnte mit Schopenhauer in der Überwindung der Schwere, der Trägheit der Materie im Raum ein Charakteristikum der Baukunst erkennen wollen. Anders als Schopenhauer gilt Nietzsche diese Überwindung jedoch als Ausdruck der Willenssteigerung und nicht etwa (wie bei Schopenhauer alle Kunst) als temporäres Willensquietiv. Während Schopenhauer der Musik den höchsten Platz einräumt, weil sie nicht bloß Ideen veranschaulicht, sondern den Willen selbst abbildet, von dessen Bedrängnis sie unter allen Künsten deshalb auch am besten (zeitweilig) zu therapieren versteht, rückt in der Götzen-Dämmerung die Architektur in diese Position auf ${ }^{14}$, gerade weil sich in ihr äußerste Willenskraft manifestiert. Nietzsche hält bekanntlich die Willensbejahung statt der künstlerischen Willenssedierung oder asketischen Willensverneinung für das Gebotene - wie hoch auch immer der Leidenstribut sein mag. Über die Qualität und den Stellenwert einer Kunst entscheidet zwar immer noch mit, in welcher Intensität sich in ihr Wille manifestiert. Ausschlaggebend ist aber nicht länger, inwiefern sie das Drängen des Willens beruhigt und Leiden verringert, sondern im Gegenteil, inwiefern sie zur Steigerung des Willens beiträgt. Jene Kunst, die die stärkste Willens-

13 Arthur Schopenhauer, Die Welt als Wille und Vorstellung I 3, § 43, in: Ders.: Sämmtliche Werke, hg. von Julius Frauenstädt, Leipzig 1873-1874, Bd. 2, 252 f.

14 Das erinnert an die Bedeutung, die die Architektur in der Renaissance gewinnt - und schon bei Vitruv, De architectura libri decem I 1, 1-18 (dazu Andreas Urs Sommer, Lexikon der imaginären philosophischen Werke, Berlin 2012, 24 f.). Der Renaissancismus ist auch in der Evokation des „grossen Stils“ (GD, KSA 6, 119) greifbar, der auf Giorgio Vasaris ,maniera grande‘ zurückverweist. 
kraft und damit die stärkste Willensbejahung zum Ausdruck bringt, hat Anspruch auf den obersten Rang - ganz gleichgültig, wie es um die mit der Willensartikulation verbundenen Leiden bestellt ist: Kunst wird keine therapeutische, sondern eine vitalisierende Funktion zugeschrieben.

Unterstreichen lässt sich dieser Befund mit der Beobachtung, dass in den Abschnitten 10 und 11 der Streifzüge eines Unzeitgemässen (sowie in den Vorarbeiten) nicht abstrakt von dieser oder jener Kunst gesprochen wird und davon, was denn deren Eigentümlichkeit sei, sondern von den jeweils ausübenden und unterschiedlichen Räuschen ergebenen Künstlern. Nicht von Architektur wird gehandelt, sondern vom Architekten als dem Willensberauschten. Der Wille selbst ist nichts Abstraktes, kein metaphysisches Weltprinzip, sondern etwas Konkretes, etwas Vielfältiges. Nicht auf eine Kunst an sich kommt es an, sondern auf konkrete Weisen der Weltgestaltung und der Weltbejahung. Indessen: Nietzsche will weder eine ideale Stufenleiter der Künste revidieren, noch hegt er selbst das Bedürfnis, Baumeister zu werden. Nähme man das bislang Ausgeführte zum Nennwert, müsste Nietzsche seinen Beruf wechseln und sich in der Baubranche versuchen. Aber offenkundig denkt er nicht daran umzuschulen.

Denn in der bisherigen Erörterung haben wir großzügig übersehen, dass der Willensrausch der Architekten ein abgeleiteter ist, seien doch „[d]ie mächtigsten Menschen“ (GD, KSA 6, 118) ihre Inspiratoren: stehen sie „unter der Suggestion der Macht“ (GD, KSA 6, 118), ist zunächst nicht ihre eigene Macht gemeint, sondern diejenige ihrer potenten Auftraggeber, die sich oder ihre Ideen in einem monumentalen Bau verewigen lassen möchten. Leicht entsteht der Eindruck, die privilegierte Stellung, die Nietzsche der Architektur im Konzert der Künste einzuräumen bereit ist, gründe nicht unwesentlich im soziologisch banalen Befund, dass die Chance der Architekten, mit politisch-ökonomischen Machtmenschen in Berührung zu kommen, ungleich größer ist als beispielsweise die von Lyrikerinnen, Aquarellisten oder Kammermusikerinnen. Träfe freilich dieser Eindruck zu, wäre die Kunst als souveräne Potenz entwertet und hätte nur noch eine (dann dafür laute) Stimme, wenn sie sich zur Dienerin der Mächtigen macht. Selbst wenn man diesen Eindruck rasch zu verscheuchen sucht, indem man darauf beharrt, dass der Architekt in den Akten seiner Weltgestaltung Macht und Machtwillen ausagiert, bleibt der Befund, dass die Baukunst keineswegs die einzige Weise ist, ,Willen zur Macht‘ auszuleben: „grosser Stil“ (GD, KSA 6, 119) ist mitnichten ein exklusives Potential der Baukunst.

Nietzsche ergreift nicht das Architektenhandwerk, sondern bleibt Philosoph, denn auch als Philosoph steht ihm der „grosse Stil“ (GD, KSA 6, 119) zu Gebote. Die Architektur bietet ihm in den Streifzügen eines Unzeitgemässen dazu kein Rollenvorbild, sondern nur eine Analogie, insofern sie ebenso wie die richtig verstandene, von allen lebensfeindlichen Hemmnissen befreite Philosophie (der Zukunft) das Antlitz der Welt tatkräftig verändern soll: Ingressivität, Weltgestaltungsstreben sind Charakteristika jenes von Architektur und (künftiger) Philosophie gleichermaßen verkörperten Machtwillens - Charakteristika, die auf anderen kreativen und intellektuellen 
Betätigungsfeldern wie der Lyrik oder der Philologie weniger ausgeprägt sind. Die Philosophie (der Zukunft) unterscheidet sich freilich von der Architektur wesentlich dadurch, dass ihr „Rausch des grossen Willens“ (GD, KSA 6, 118) sich nicht abhängig macht von solchen, die über die ökonomischen und politischen Mittel verfügen, einen Architekten erst mit einem Bauwerk zu beauftragen: Der Philosoph (der Zukunft) ist selbstmächtig, während der Architekt auf fremde Macht angewiesen bleibt.

Die Analogie zwischen Architektur und Philosophie funktioniert demnach nur partiell; sie dient als Metapher für Philosophie, für den philosophischen Willensrausch und für das philosophische Weltgestaltungsstreben. In der Charakterisierung des Architekten tritt das Produkt seines Tuns zurück, bis auf den Satz, darin solle „sich der Stolz, der Sieg über die Schwere, der Wille zur Macht versichtbaren“ (GD, KSA 6, 118). Das Bauwerk steht aber nicht im Vordergrund; Baukunst interessiert nicht so sehr als Poiesis, als Hervorbringung, sondern als Praxis, als Tun, das seinen Zweck in sich selbst hat, nämlich als willensberauschte Machtpraxis. Auch hier wäre die Übertragbarkeit auf Nietzsches Unternehmung einer Zukunftsphilosophie zumindest zu diskutieren. In offensichtlichem Gegensatz steht der Akzent auf Architektur als Praxis jedenfalls zum landläufigen Gebrauch der Wissensarchitekturmetapher, bei der das Hervorgebrachte, das Bauwerk und nicht etwa die Weisen der Hervorbringung im Zentrum stehen. Wer Wissen wie ein Gebäude organisiert haben will, mag sogar dazu tendieren, die Weisen der Hervorbringung dieses Gebäudes als schöpferische Akte unsichtbar zu machen: Das Wissensgebäude soll ja nicht den Anschein der Beliebigkeit und schöpferischen Willkür erwecken, sondern - alle Tätigkeit der Wissensordnung und Wissensorganisation verschleiernd - so erscheinen, als ob es Abbild der Wirklichkeit selbst wäre, Abbild dessen, wovon es Wissen ist. Dass es eine menschlich-künstlerische Praxis ist, die die Paläste (und Hütten) des Wissens erst hervorbringt, blendet derjenige gerne aus, der sich als Palasthüter versteht. Neben ihm wirkt Nietzsche subversiv, wenn er sich der Architektur als Metapher für kreative Denkpraxis bedient.

\section{Nietzsche als Abbruchunternehmer großen Stils}

„Grosser Stil“ (GD, KSA 6, 119) ist ein wesentliches Stichwort, unter dem Nietzsche in den Streifzügen eines Unzeitgemässen das Tun des Architekten verhandelt. Er sucht großen Stil allerdings keineswegs nur oder hauptsächlich in der Baukunst; vielmehr ist ihm dieser große Stil Maßgabe künstlerischen, intellektuellen, ja überhaupt weltgestalterischen Tuns. Gewiss drückt sich darin eine (durchaus zeittypische) Bewunderung für das Kolossale, Gigantische, aber zugleich auch für das Festgefügte, Horizontbestimmende, Obdachgebende aus. Nietzsches positive Urteile über vergangene Kulturen, beispielsweise über das vorsokratische Griechenland, die Manu-Gesetzesordnung oder die Renaissance, sind formuliert als Urteile über Epochen großen Stils, 
den er in seiner Gegenwart schmerzlich vermisst. Das Römische Reich etwa, das in Nietzsches Spätwerk zu unverhoffter Ehre kommt, erscheint als das „bewunderungswürdigste Kunstwerk des grossen Stils“, dessen „Bau“ „berechnet“ war, „sich mit Jahrtausenden zu beweisen, - es ist bis heute nie so gebaut, nie auch nur geträumt worden, in gleichem Maasse sub specie aeterni zu bauen!“ (AC, KSA 6, 246) Für den großen Stil bieten sich also nach wie vor architektonische Metaphorisierungen an bemerkenswert übrigens die Parallelisierung von Bauen und Träumen! - , und die, die diesem Staats- und Gesellschaftsbauwerk gemäß Antichrist gefährlich werden, nämlich die Christen, sind ausdrücklich jene, die Hand ans Fundament legen, die es untergraben und ruinieren.

Nietzsches Sehnsucht nach dem großen Stil ist eine Sehnsucht, selbst Bleibendes, Abgeschlossenes, Gerundetes zu schaffen. Diese Sehnsucht findet Nahrung in den diversen Entwürfen zur systematischen Auftürmung seiner Philosophie, die in den Manuskriptheften hinterlassen sind. Bekanntlich ist Nietzsche diese Ausarbeitung, das große Bauwerk, versagt - oder erspart geblieben. Umso eifriger bemühen sich die Verwalter seiner Hinterlassenschaften - angefangen mit Elisabeth Förster bis hin zu seinen jüngsten angloamerikanischen Exegeten - , darum, die systemische Geschlossenheit in das überlieferte Corpus seines Schreibens zu implantieren: Während die Schwester mit ihren Nachlass-Kompilationen, insbesondere dem Willen zur Macht, durch Anreicherung und Arrangement dessen, was ursprünglich (gar nicht) da war, den äußeren Anschein der systemischen Schließung zu erzeugen suchte, verfahren die neuesten Exegeten in ähnlicher Absicht so, indem sie das überlieferte Corpus einer rigorosen Politik der Weglassung und Ausblendung unterwerfen. Jüngstes anschauliches Beispiel dafür gibt der Versuch, Nietzsche als ,Naturalisten“ $\mathrm{zu}$ vereinnahmen und über seinen, Naturalismus` zu streiten, als ob sich dieser so wendige Autor irgendwie in den grobschlächtigen Kategorien der Nachkriegs-Philosophieverwaltungswissenschaft unterbringen ließe. ${ }^{15}$ Das ist nur einer aus einer Fülle von Versuchen, Nietzsche gefügig, häuslich zu machen. Die philosophisch ambitionierten Nietzsche-Interpreten waren von Anbeginn an eifrig darum bemüht, einen Grundriss ins wüste Mischmasch von Nietzsches Schreibwerkstatt hineinzubauen, ihm etwa eine Architektur des Willens zur Macht oder der Ewigen Wiederkunft oder des Übermenschen als ,Hauptlehren“ anzudichten. ${ }^{16}$ Dabei gebärden sie sich wie Denkmalpfleger, die bauen, was gar nie da war, aber hätte da sein sollen, sozusagen als die Viollet-le-Ducs der Philosophie, mit einer einzigen Perspektive: Nietzsche im Gehäuse zu hegen, ihn handhabbar, handzahm zu machen.

15 Vgl. den kritischen Überblick bei Dirk Johnson, New Anglo-American Anthologies on Nietzsche, in: Nietzsche-Studien 43 (2014), 229-240.

16 Siehe Andreas Urs Sommer, Nietzsche katalytisch. Philosophische Nietzsche-Lektüren im 20. Jahrhundert, in: Marco Brusotti, Renate Reschke (Hg.), „Einige werden posthum geboren“. Friedrich Nietzsches Wirkungen, Berlin, Boston 2012, 21-50. 
Damit antworten diese Interpreten durchaus auf Nietzsches eigenes Bemühen, sein Denken architektonisch zu fundieren. Aber bei diesen Versuchen ist ihm und ihnen dieses Denken immer wieder entglitten, denn offensichtlich ist der dominierende Modus dieses Denkens die Verflüssigung und nicht das Konsolidieren. Unentwegt fällt sich Nietzsche selbst ins Wort; er ist der Denker der permanenten Mobilisierung, unentwegt auf der Suche nicht nur nach neuen Ausdrucksformen, sondern auch nach neuen Denkinhalten. Seine Polemik gegen philosophischen Systembau ist fast schon legendär - sie richtet sich gegen die Einigelung in intellektuellen Gehäusen, gegen die Horizontverschließungen, will frei machen fürs Hochgebirge. Es ist ein Philosophieren in fortgesetzter Selbstaufhebung. So viel Gefallen Nietzsche an architektonischen Metaphern auch findet: Sein Denken stößt Stabilisierung ab; es sträubt sich gegen Erstarrung und bleibt beweglich.

Wer als Interpret trotzdem seiner Vorliebe für Lehren nicht abschwören will, wird argumentieren, in dieser permanenten Mobilisierung manifestiere sich das, was Nietzsche ,Wille zur Macht' nenne. Diese Argumentation wird pointieren, dass der Willensrausch nicht, wie in der Götzen-Dämmerung behauptet, vor allem in der architektonischen Gestaltung seine Erfüllung finde, sondern für Nietzsche selbst doch ebenso sehr im Zerstören, im Zerstören des vermeintlich Großen und Größten. Sicher, Nietzsche pflegte stets zu betonen, dass die Verneinung von der Bejahung übertrumpft werden solle, und dass die Verneinung nur ein Mittel zur Bejahung sei. Dennoch münze sich die Bejahung trotz aller pathetischen Gesten faktisch nicht in Bejahung aus; Nietzsche erwecke zwar die Suggestion des Bauens, bleibe aber im Grunde destruktiv. Im Grunde: Das Zerstören diene dem Machtwillen und dem Willensrausch mehr als das mühsame Aufbauen; in der Destruktion gewinne man der Macht, dem Willen und dem Rausch sattere Farben und ein reicheres Geschmacksspektrum ab als in der Konstruktion. Kein Wunder also, dass sich der willensrauschlüsterne Philosoph entgegen aller Lippenbekenntnisse vornehmlich der Verneinung verschrieben habe.

Manchem wird diese ad hoc-Rekonstruktion von Nietzsches Denkbewegung gewaltsam und denunziatorisch vorkommen, legt sie ihn doch fest auf einen einzigen dominanten Gedanken, den Willen zur Macht, den sie als ein Prinzip interpretiert, das in der Destruktion viel intensiver erlebt werden kann als in der Konstruktion, was immer sein Erfinder an Gegenteiligem behaupten mag. Nun ist es gewiss richtig, dass Nietzsche den Willen zur Macht wenigstens in seinen letzten Schaffensjahren als Markenzeichen wie eine Monstranz seiner Denker-persona voranträgt. Nietzsches Machtwille ist maximaler Wirkungswille. Aber Monstranz und Markenzeichen verdecken vieles - vor allem vieles, was sich nicht auf das angeblich Eine, den Willen zur Macht reduzieren lässt. Nietzsches Denkgebäude ist kein in sich stimmiges, austariertes und wohlproportioniertes Bauwerk, fundamentiert oder bekrönt vom Willen zur Macht. Wer den Blick über sein Werk schweifen lässt, wird stattdessen eher eine Ruinenlandschaft vorfinden, bestückt mit den halb von selbst verfallenen, halb der Abrissbirne zum Opfer gefallenen Systembauwerken des abendländischen Denkhaushalts. Und 
der Herr über diese Ruinenlandschaft ist keineswegs geneigt, sie romantisch zu verklären. Gelegentlich versucht er sich an Neubauten, gelegentlich sogar an Restaurationsarbeiten (bei Archaischem notabene), meist aber bleibt er in Bewegung, verschmäht das gemauerte Fundament, bevorzugt - nomadisch - das Zelt oder besser noch den freien Himmel.

Dabei erweist er sich als exemplarischer philosophischer Situationist, der auf Gegebenes antwortet. Jenseits seiner Setzungen, an denen heutige Interpreten festzukleben pflegen, übt er ein Denken in Oppositionen - ein Denken, das reagiert auf ein Behauptetes oder Geglaubtes, um Widerspruch durch Negation anzumelden. Nietzsche ist bestimmt von einem geradezu zwanghaften Andersseinwollen, das sich mit dem Anspruch verbindet, nur dieses Anderssein sei wahrhaft bejahend. Nietzsches Kraft ist die Kraft der Verstörung. Diese Kraft ist zwar auch zu guten Stücken Kraftmeierei - was aber nichts an der Wirkmächtigkeit dieser Kraft ändert.

Nietzsches Denken ist hochgradig instabil, es lässt tausend Hintertüren jeder möglichen Interpretation und Adaption offen. Sein anhaltendes Erregungspotential liegt darin, dass er immer wieder neu anfängt, dass seine Denkbewegung kein Kontinuum ist, sondern ein stetes Abbrechen und ein stetes Neubeginnen. Vor hundert Jahren hätte man gesagt, darin entspreche er genau der Nervosität des Zeitalters. Multiperspektivität ist bei Nietzsche nicht einfach nur verlangt und postuliert, sondern praktiziert. Das lädt die Leser ein, ihn ihren Perspektiven anzupassen. Offenheit und Extremismus sind die Zaubermittel dieses Schreib-Denkens. Solches vorbehaltvolles Schreib-Denken entspricht so gar nicht dem vorherrschenden Comment des assertorischen Philosophen-Indikativs. Wenn Nietzsche etwas baut, dann Labyrinthe.

Und doch schreibt er mit dem Anspruch, etwas Bleibendes zu schaffen. Um diesen Anspruch zu untermauern, zitiert er mit Vorliebe einen Vers, der in seiner vollständigen Form aufs Architektonische Bezug nimmt: „Man wird, bis in meinen Zarathustra hinein, eine sehr ernsthafte Ambition nach römischem Stil, nach dem , aere perennius im Stil bei mir wiedererkennen“ (GD, KSA 6, 154). „Exegi monumentum aere perennius / regalique situ pyramidum altius“ („Ich habe ein Denkmal errichtet, beständiger als Erz / höher als der königliche Sitz der Pyramiden“), schreibt Horaz ${ }^{17}$ und prophezeit damit seiner eigenen Dichtung eine Zukunft, die physisch-menschlichen Kulturerrungenschaften, selbst die mächtigsten Bauwerke überdauern wird. Nietzsche zitiert jeweils nur zwei lateinische Worte, „aere perennius“ („,beständiger als Erz“) (GD, KSA 6, 154), kann er bei den Gebildeten unter seinen Lesern doch voraussetzen, dass ihnen das Original geläufig ist. Das wirklich Beständige ist weder aus Metall noch von Menschenhand aus Stein aufgetürmt, vielmehr ist es in Schrift gegossen. Das wirklich Beständige ist kein Produkt des ob seines Willensrausches so gerühmten Architekten. Dennoch bleibt der implizite Maßstab für die eigene Unvergänglichkeit und Größe ein Bauwerk, die ägyptischen Pyramiden. Jedoch verschweigt Nietzsche sie, um so die

17 Quintus Horatius Flaccus, Carmina III 30, $1 \mathrm{f}$. 
Macht der architektonischen Metapher nicht erneut heraufzubeschwören, während Horaz durch ihre Nennung die Macht der Architektur ausdrücklich in Schranken weist. Nietzsche beschränkt sich auf das korrosionsanfällige Erz als Vergleichsgröße. Dem Erz ist das Quecksilber seines Denkens allemal überlegen. 